\title{
Complete lung agenesis caused by complex genomic rearrangements with neo-TAD formation at the SHH locus
}

\author{
Uirá Souto Melo ${ }^{1,2} \cdot$ Juliette Piard $^{3} \cdot$ Björn Fischer-Zirnsak ${ }^{2} \cdot$ Marius-Konstantin Klever $^{1,2} \cdot$ Robert Schöpflin $^{1}$. \\ Martin Atta Mensah ${ }^{2,4}$. Manuel Holtgrewe ${ }^{4}$. Francine Arbez-Gindre ${ }^{5}$ - Alain Martin ${ }^{5}$. Virginie Guigue ${ }^{5,6}$. \\ Dominique Gaillard $^{6} \cdot$ Emilie Landais $^{6} \cdot$ Virginie Roze $^{5} \cdot$ Valerie Kremer $^{7,8,13} \cdot$ Rajeev Ramanah $^{5} \cdot$ Christelle Cabrol $^{3}$. \\ Frederike L. Harms ${ }^{9} \cdot$ Uwe Kornak $^{10}$ • Malte Spielmann ${ }^{1,11}$. Stefan Mundlos ${ }^{1,2} \cdot$ Lionel Van Maldergem $^{3,4,12}$
}

Received: 3 June 2021 / Accepted: 10 August 2021 / Published online: 26 August 2021

(c) The Author(s) 2021

\begin{abstract}
During human organogenesis, lung development is a timely and tightly regulated developmental process under the control of a large number of signaling molecules. Understanding how genetic variants can disturb normal lung development causing different lung malformations is a major goal for dissecting molecular mechanisms during embryogenesis. Here, through exome sequencing (ES), array CGH, genome sequencing (GS) and $\mathrm{Hi}-\mathrm{C}$, we aimed at elucidating the molecular basis of bilateral isolated lung agenesis in three fetuses born to a non-consanguineous family. We detected a complex genomic rearrangement containing duplicated, triplicated and deleted fragments involving the SHH locus in fetuses presenting complete agenesis of both lungs and near-complete agenesis of the trachea, diagnosed by ultrasound screening and confirmed at autopsy following termination. The rearrangement did not include $S H H$ itself, but several regulatory elements for lung development, such as MACS1, a major $S H H$ lung enhancer, and the neighboring genes $M N X 1$ and $N O M 1$. The rearrangement incorporated parts of two topologically associating domains (TADs) including their boundaries. Hi-C of cells from one of the affected fetuses showed the formation of two novel TADs each containing SHH enhancers and the MNXI and NOMI genes. Hi-C together with GS indicate that the new 3D conformation is likely causative for this condition by an inappropriate activation of MNXI included in the neo-TADs by MACS1 enhancer, further highlighting the importance of the 3D chromatin conformation in human disease.
\end{abstract}

\section{Introduction}

Complete absence of one or both lungs is an extremely rare malformation with an estimated birth prevalence below $1 / 10^{7}$ if we estimate that only a dozen of reports have been published so far, mostly occurring unilaterally (Ostör et al. 1978; Mardini et al. 1985; Spear et al. 1987; Engellenner et al. 1989; Podlech et al. 1995; Kayemba-Kay's et al. 2014). This extreme deleterious phenotype is caused by a failure in the proper formation of the lung buds derived from the foregut with no detectable respiratory tissue (lung agenesis)

Uirá Souto Melo and Juliette Piard contributed equally to this work.

Deceased to the contact information of Lionel Van Maldergren.

Stefan Mundlos

stefan.mundlos@charite.de

Extended author information available on the last page of the article or by their deficient proliferation and branching leading to the development of short, blind ending bronchi (lung aplasia) during the early embryonic stage. Understanding how genetic variants can cause different lung malformations is a major goal for dissecting molecular mechanisms during embryogenesis.

During lung development, the conducting airways are formed first, followed by the formation of alveolae. The left and right lungs have their own anlage, derived from the anterior foregut endoderm, a tissue where also thyroid, esophagus, and liver are originated from (Kadzik and Morrisey 2012). In humans, in the late 4th week after conception, the embryonic stage of lung development starts with the formation of two outpouchings in the ventral wall of the foregut termed lung buds, that start to proliferate in repetitive circles of growth and branching (Schittny 2017). While these buds, that form the later respiratory epithelium, are derived from the endoderm, they grow and branch inside of mesoderm derived tissues. An intensive 
crosstalk between endodermal and mesenchymal mesodermal cells is required for the formation and growth of the lung buds. This crosstalk also regulates the simultaneous development of the mesoderm derived cardiopulmonary vasculature together with the respiratory epithelium (Schittny 2017; Kimura and Deutsch 2007; Swarr and Morrisey 2015).

Here we evaluate a family with three fetuses affected by complete lung agenesis suggestive of an autosomal recessive inheritance pattern. Combining array $\mathrm{CGH}$ with genome sequencing (GS) and chromosome conformation capture (Hi-C) data, we were able to dissect this case in its complexity. We identified a complex rearrangement at the SHH locus that is proposed to result in the extreme lung condition in our cases. To the best of our knowledge, no study has been done to interrogate the molecular cause of isolated lung agenesis in humans.

\section{Methods}

\section{Subjects, collected samples and cell culture}

Healthy parents provided written informed consent to all subjects enrolled in this study. All biopsies and molecular testing were performed after obtaining written consent of the patients in accordance with the rules of Helsinki. Blood samples were collected from grandparents (I-1 and I-2), healthy parents (II- 1 and II-2) and one healthy sibling (III2 ). We collected fetal material from three affected individuals (III-1, III-3 and III-4; Fig. 1a).

Fibroblast cell lines were established from skin biopsies of the father (II-1) and the Fetus 2 (III-3). Fibroblasts were cultured in DMEM (Thermo Fisher Scientific) supplemented with $10 \%$ fetal bovine serum (FBS; Thermo Fisher Scientific), 1\% L-glutamine (Thermo Fisher Scientific) and $1 \%$ penicillin-streptomycin (Thermo Fisher Scientific).
Fig. 1 Family tree and phenotypes of the three affected fetuses. a Family pedigree. DNA samples were available from individuals marked with blue asterisk (*) and fibroblast from those marked with green number symbol (\#). b Autopsy of Fetus 1 shows absence of the lungs. White arrows indicate a very small bud of lung. White asterisk indicates the location where trachea should have been observed. Other organs are present. c Autopsy of Fetus 2 showing lung agenesis. Gallbladder was absent. d Similar to Fetus 1, empty thorax is observed in Fetuses 2 and 3 a

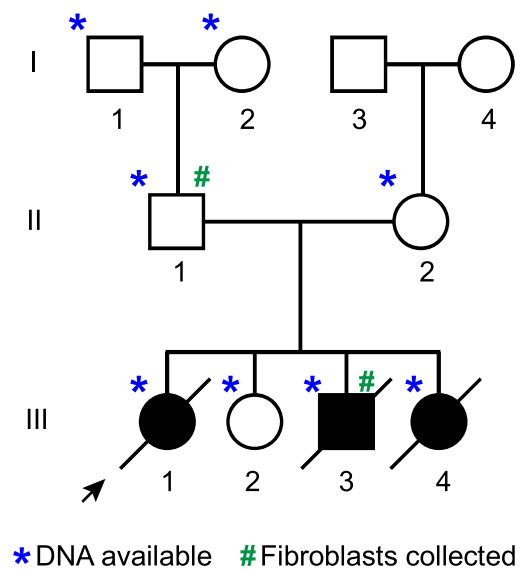

C

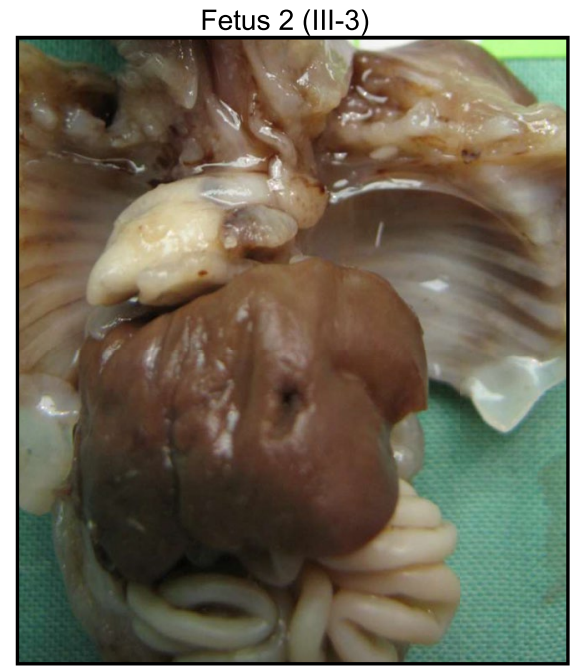

b

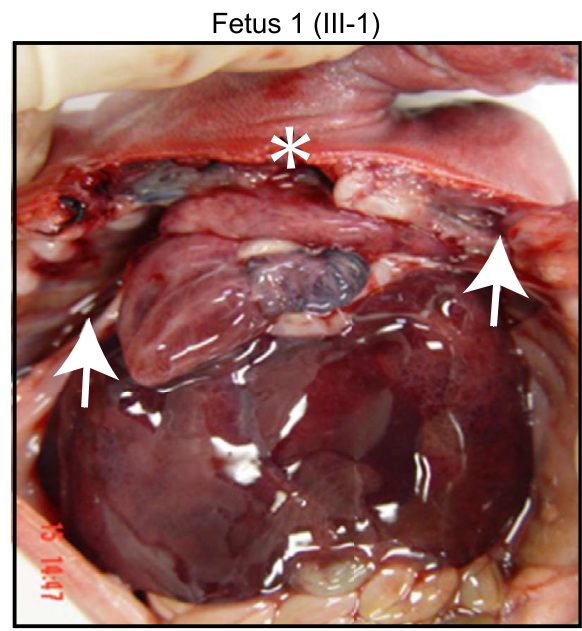

d

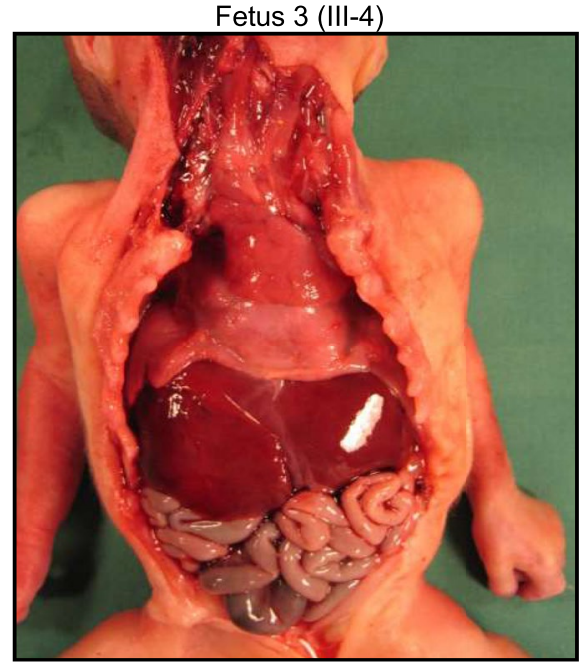


Fibroblasts from two unrelated healthy individuals were used as controls.

\section{DNA and RNA extraction, and quantitative PCR analyses (qPCR and RT-qPCR)}

DNA and RNA were extracted using DNeasy Blood and Tissue Kit (Qiagen) and RNeasy Mini Kit (Qiagen), respectively. qPCR was performed to measure the copy number of fragments detected by array CGH. RT-qPCR was performed to measure the expression of candidate genes located at the SHH locus: SHH, RNF32, LMBR1, NOM1, MNX1 and $U B E 3 C$. Primer sequences are available upon request. qPCR and RT-qPCR were performed using the PowerUp ${ }^{\mathrm{TM}}$ SYBR ${ }^{\circledR}$ Green Master Mix (Thermo Fisher) in the QuantStudio 6 Flex Real-Time PCR System, 384-well (Applied Biosystems). Copy number and gene expression were calculated using $2^{-\Delta \Delta \mathrm{CT}}$ method (Schmittgen and Livak 2008). Each experiment was performed once with three technical replicates per sample.

\section{Genomic screening}

Microarray-based comparative genomic hybridization (array $\mathrm{CGH}$ ) was performed in DNA from the healthy father (II-1; blood) and two fetuses (III-1 and III-3) using the $1 \mathrm{M}$ arrays (Agilent, Santa Clara, CA). Copy number variation (CNV) detected by array CGH were further assessed by qPCR of the following fragments: A (15 Kb duplication, chr7:156 ,181,014-156,196,660×3); B (449 kb triplication, chr7:1 $56,196,660-156,645,844 \times 4)$; C (207 kb duplication, chr 7:156,645,844-156,853,796×3), D (neutral copy, $9.8 \mathrm{~kb}$, chr7:156,853,796-156,863,687) and $\mathrm{E}(61 \mathrm{~kb}$ deletion, chr 7:156,863,687-156,924,827×1), also calibrator regions A' and E', located up- and downstream the rearrangement.

Targeted enrichment and massively parallel sequencing were performed on genomic DNA from the healthy father (blood) and two fetuses (III-1 and III-3). Enrichment of the Exome was performed according to the manufacturer's protocols using the Nextera Enrichment Kit (62 Mb) (Illumina). Captured libraries were then loaded onto the 2500 platform (Illumina). The following genes referenced in OMIM as associated to agenesis, aplasia or hypoplasia of the lung in humans were analyzed for rare, likely pathogenic variants: FOXF1 (\#265380); ZFPM2, TBX4 and FGF10 (\#265430).

Genome sequencing was performed in the healthy father (blood) and Fetus 1 (III-1) to validate the CNVs detected by array CGH and to identify the breakpoints at the base pair level to disentangle the nested complex rearrangement. Details of the protocol and pipeline are described previously (Melo et al. 2020).

\section{Hi-C analysis}

Hi-C libraries were processed with our in-house pipeline based on the previously published in situ protocol (Rao et al. 2014). Briefly, 1 million cells were fixed in $2 \%$ formaldehyde, lysed and digested overnight with DpnII enzyme (New England BioLabs). Next, digested DNA ends were marked with biotin-14-dATP (Thermo Fisher Scientific) and ligated overnight using T4 DNA ligase (New England BioLabs). DNA was sheared to fragments of 300-500 bp for library preparation and biotin-filled DNA fragments were pulled down using Dynabeads MyOne Streptavidin T1 beads (Thermo Fisher Scientific). The DNA was prepared for short reads sequencing by ligating adaptors to the DNA fragments, using the NEBNext Multiplex Oligos for Illumina kit (New England BioLabs). Libraries were deep sequenced ( 240 Million fragments) in a 75 bp paired-end run on a HiSeq4000 (Illumina). Paired-end sequencing data were processed using the Juicer pipeline (Durand et al. 2016). A detailed protocol is described elsewhere (Melo et al. 2020).

\section{Results}

\section{Clinical evaluation and autopsies of three fetuses revealed complete bilateral lung agenesis}

The first pregnancy of a Caucasian woman aged 25 (II-2), unrelated to her 27-year-old partner (II-1), was remarkable for the detection of complete bilateral lung agenesis by ultrasound during the 2nd trimester of pregnancy (Fig. 1a and b). The next three pregnancies disclosed one healthy girl (III-2) and two other fetuses, a male and a female, also presenting the same severe lung phenotype.

The first fetus, a female (III-1), examined after termination of the pregnancy at 22 weeks indicated growth parameters within the normal range $(510 \mathrm{~g})$. Autopsy of the thorax revealed complete absence of the lung tissue (Fig. 1b). The heart was medially displaced and did not present any malformation. A complete atresia of a shortened trachea was observed, its distal part being measured at $5 \mathrm{~mm}$ of laryngeal structures. Left and right pulmonary artery were absent. There was no other malformation of the viscera, face or limbs. Histology of thymus and esophagus were normal.

The second fetus, a male (III-3), was examined after termination of the pregnancy at 24 weeks. His weight was $710 \mathrm{~g}$ (mean). Autopsy of the thorax revealed neither lung nor trachea in this fetus (Fig. 1c). Crown-rump distance was $22.5 \mathrm{~cm}$ (NR). $2 \mathrm{~mm}$ under the larynx, a small dimple in a structure that resembles a tracheal bud is seen without any lumen. Similar to Fetus 1 (III-1), integrity of the trunk of the pulmonary artery was observed while its branches were absent. There was no pulmonary vein return in the 
left atrium. Penis length was at lower limit of normal range $(9 \mathrm{~mm})$ and the gallbladder was absent. Histology was unremarkable.

The third fetus, a female (III-4) was examined after termination at 17 weeks of gestation. This fetus had normal growth parameters and similar autopsy findings with the other two fetuses (Fig. 1c).

Complete lung agenesis is an ultra-rare event and led us to propose a genetic cause of recurrence in the three evaluated conceptuses.

\section{Genetic screening revealed a complex rearrangement on $7 q 36.3$ in all three fetuses}

Chromosomal anomalies were ruled out by performing standard karyotyping in both parents and in the three fetuses (data not shown). Based on the pedigree (Fig. 1a), an autosomal recessive mode of inheritance was first taken into consideration. We performed exome sequencing (ES) in Fetuses 1 and 2 and searched for mono- or biallelic rare variants in known genes related to agenesis, aplasia or hypoplasia of the lung in humans (see "Methods") and in the orthologue genes in mouse (ALDH1A2, FGFR2, TCF21, BCLAF1, MEK1 and MEK2) (Park et al. 2019; Arman et al. 1999; McPherson et al. 2009; Wang et al. 2006; Boucherat et al. 2014). ES failed to identify potential deleterious variants in homozygosis or compound heterozygosis in these genes. Furthermore, no other potential candidates neither recessive, nor dominant de novo were identified.

Next, we performed array CGH in blood samples from the healthy father and in fetal DNA from Fetuses 1 and 2. Although no homozygous CNVs were identified in these samples, we detected a complex genomic rearrangement (CGR) on 7q36.3 shared by both fetuses, containing duplicated, triplicated and deleted fragments (Fig. 2a). Since this structural variant (SV) is located $\sim 580 \mathrm{~kb}$ downstream of $\mathrm{SHH}$, we suspected a long-range effect on this gene implicated in lung bud development being causative. Shh is involved in lung development and a knockout mouse model for Shh exhibits lung malformations (Chiang et al. 1996; Litingtung et al. 1998). This variant was also detected in the genitor, but it showed a distinct pattern in the array CGH in comparison to the fetuses (Fig. 2a). Given a less than $1.5 \times$ copy number gain for the region containing the RNF32 gene, we assume a mosaic status of the duplication in the father.

For clarity, we named the up-( $\left.\mathrm{A}^{\prime}\right)$ and the downstream $\left(\mathrm{E}^{\prime}\right)$ copy neutral regions surrounding the $\mathrm{SV}$, as well as five fragments (A-E) defined according to their copy number value. The $\mathrm{B}$ and $\mathrm{C}$ fragments contain the RNF32, LMBRI, $N O M 1$ and $M N X 1$ genes, while the remaining fragments do not contain known protein coding genes (Fig. 2a). qPCR confirmed the findings from array CGH. Thus, the CGR consists of a duplication of the $\mathrm{A}$ and $\mathrm{C}$ fragments $(\sim 1.5 \times)$, a triplication of $\mathrm{B}(\sim 1.75 \times)$ and a deletion of $\mathrm{E}(\sim 0.5 \times)$ in all three of the fetuses' samples (Fig. 2b). qPCR performed in blood samples from the father detected $\sim 1.2$ copies of $\mathrm{A}$ and no extra copy of B (Fig. 2c) and the $\mathrm{C}$ and $\mathrm{D}$ fragments were partially reduced ( $\sim 0.85$ and $\sim 0.7 \times$, respectively), suggesting that not only just a fraction of cells harbors the complex rearrangement, but also a third allele without (C), (D) and (E) fragments is very likely to be present in the father's samples (Fig. S1c). DNA from the father's fibroblasts showed a duplication of $\mathrm{B}(\sim 1.5 \times)$ and a reduction of $\mathrm{C}(\sim 0.67 \times)$ and $\mathrm{D}(\sim 0.56 \times)$ (Fig. 2c), thus corroborating a mosaic status. The $\mathrm{E}$ fragment is deleted in heterozygous state $(\sim 0.5 \times)$ in both, the father's blood and the fibroblasts samples. The remaining healthy relatives do not carry this variant (Fig. S1a). These results suggest that the father is likely mosaic for the A-D fragments, resulting in a lower copy number state for theses fragments, providing a possible explanation for the absence of a clinical phenotype.

\section{Expression analysis in fibroblast from one affected case, the healthy father and controls}

We investigated the impact of the SV on gene expression in the available fibroblasts samples. $M N X I$ and $S H H$ are not expressed in this cell line according to our in-house fibroblast expression database; indeed, RT-qPCR did not detect both genes transcripts in all tested samples (data not shown). RT-qPCR from Fetus 2 revealed upregulation of $R N F 32(\sim 4 \times)$ and LMBRI $(\sim 1.5 \times)$ (Fig. S1b). In the healthy father, we observed reduced $L M B R 1$ and NOMI expression, which correlates with absence of these genes in one allele (Fig. 2c; Figs. S1b and c). The two candidate genes showing differential gene expression in fibroblasts were RNF32, encoding a ring finger protein involved in protein-DNA or protein-protein interactions and thought to play a role in spermatogenesis (van Baren et al. 2002); and LMBRI, a widely expressed gene with putative membrane receptor function. Our interpretation is that gene dosage of both genes is unlikely to be involved in lung agenesis. Therefore, we suspected that ectopic gene expression due to positional effect (e.g., enhancer adoption) could be the pathomechanism involved in this disease.

\section{Genome sequencing alone is not able to fully reconstruct the $\operatorname{der}(7 q)$ linear sequence}

We performed genome sequencing (GS) in samples from the healthy father (blood) and Fetus 2 to identify the breakpoints at the base pair level and solve the nested structure of the SV. We visually inspected the data for split-reads/chimeric read-pairs on 7q36.3 in both samples and observed breakpoints spanning reads for the $\mathrm{D}-\mathrm{A}$, 
a

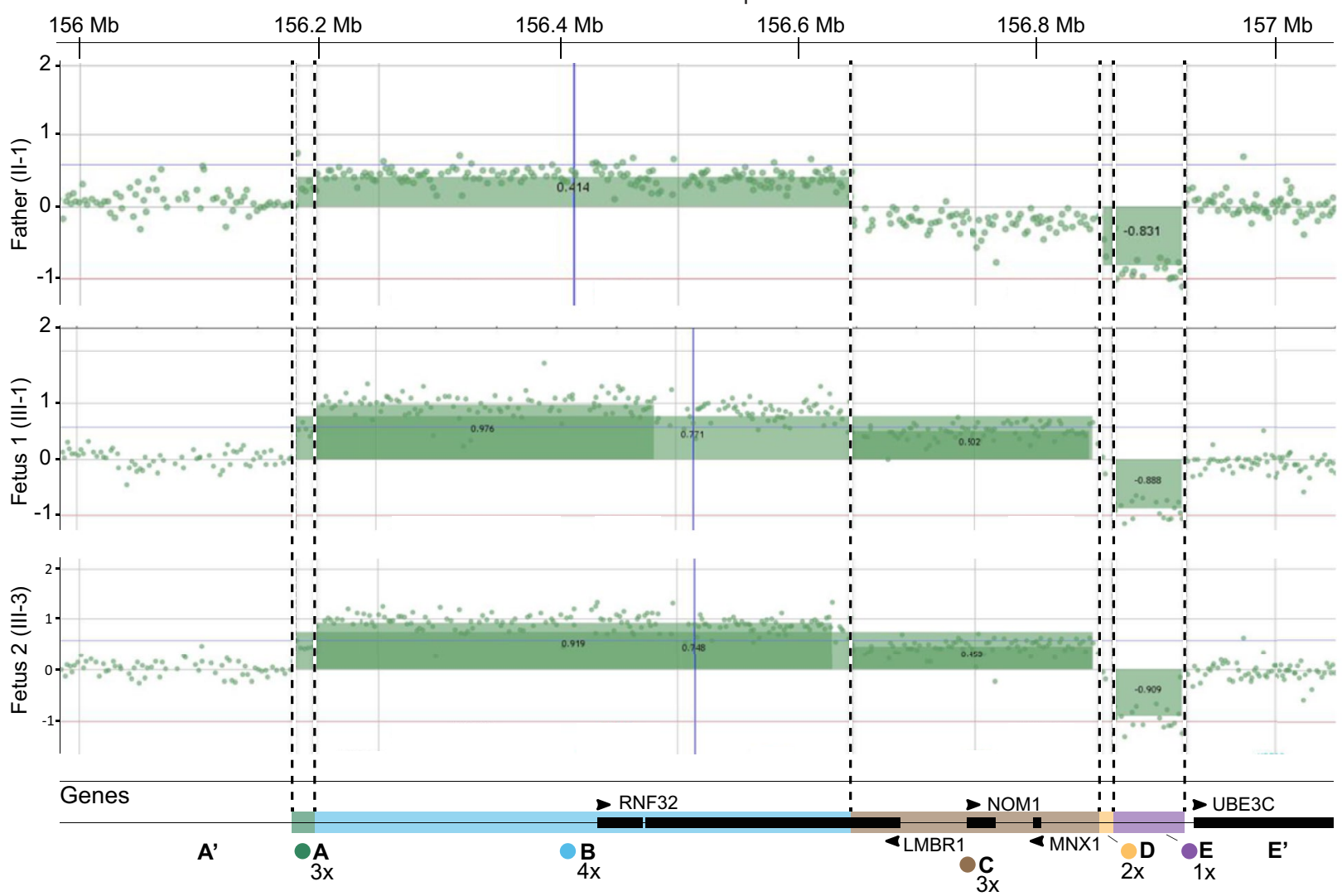

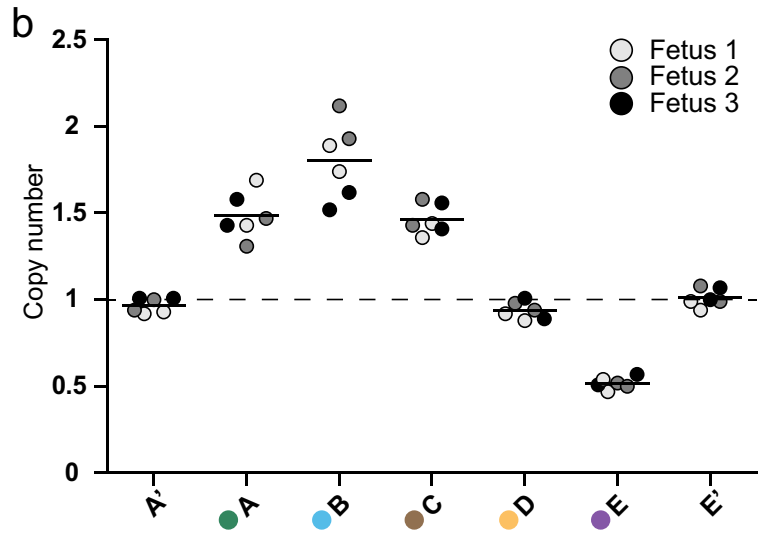

Fig. 2 Copy number variation at the 7q36.3 locus. a Array CGH detected a complex rearrangement involving duplication, triplication and deletion in two fetuses. This variant is also presented in mosaic state in the father. The fragments A-E have different copy number values: A ( $3 \times$; green), B ( $4 \times$; blue), C ( $3 \times$; brown), D ( $2 \times$; orange) and $\mathrm{E}(1 \times$; purple). $\mathbf{b}$ qPCR in fibroblasts confirmed the copy number

C-B and B-E' fragments (Figs. 3a and b). Note that few split-reads support the presence of the A-D fragments in the father, thus once again supporting mosaicism (Fig. S3). Based on GS data, the rearranged linear sequence could

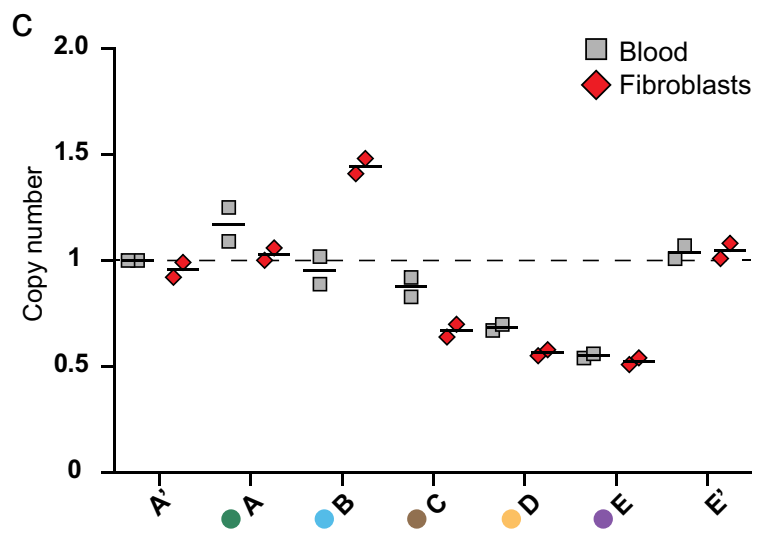

values in all three fetuses. $\mathrm{A}^{\prime}$ and $\mathrm{E}^{\prime}$ fragments are copy number neutral up- and downstream of the complex rearrangement and were used as control. c qPCR in blood (grey squares) and fibroblasts (red $45^{\circ}$ squares) samples from the healthy father showed different copy numbers for the A-D fragments, suggesting mosaicism. The $\mathrm{E}$ fragment is deleted in heterozygosis in both father's blood and fibroblasts samples

be reconstructed according to two hypothetical scenarios (i.e., Scenario 1 and 2; Fig. $3 \mathrm{~b}$ and Fig. S2). Thus, the GS split-read analysis alone is not able to fully resolve the correct linear sequence of this CGR. 
a
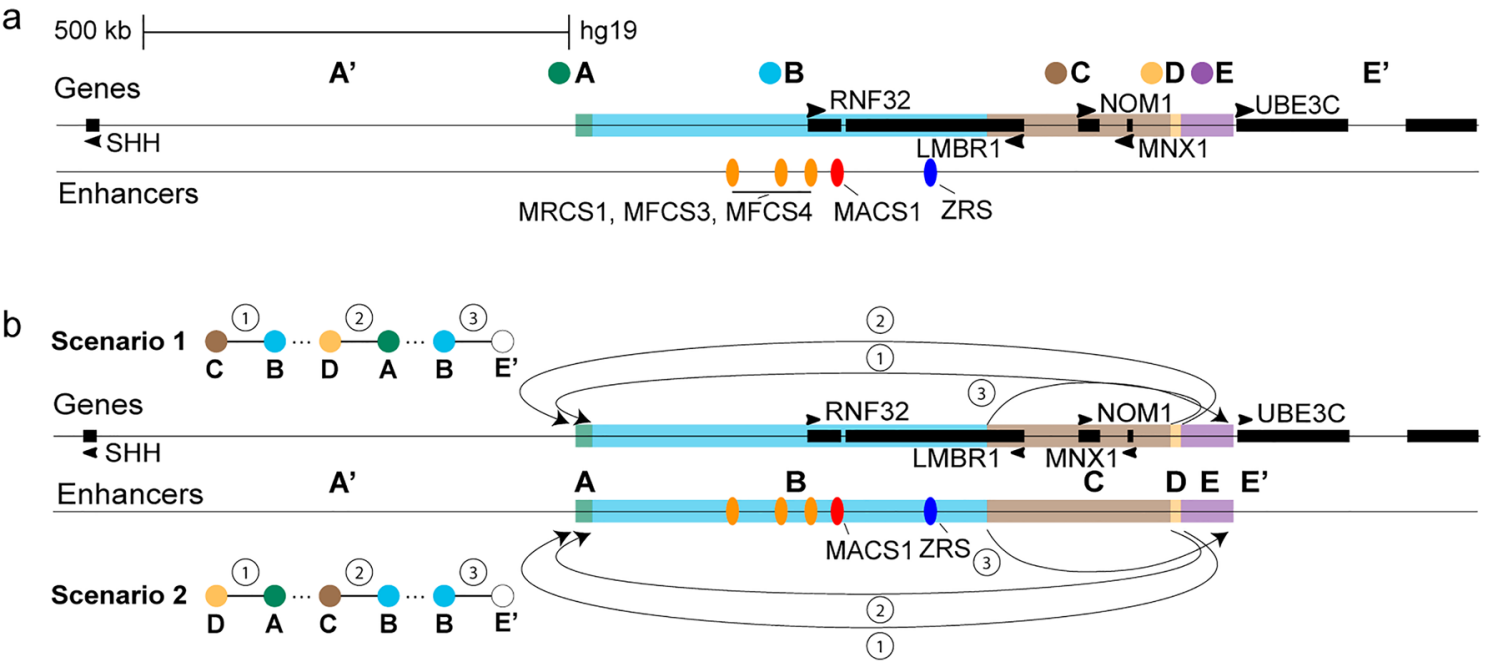

Fig. 3 Genes and enhancers at the $7 \mathrm{q} 36.3$ region. a The A and D fragments have 15 and $9.8 \mathrm{~kb}$ length, respectively, and contain neither genes nor enhancers; the same is observed for the $\mathrm{E}$ fragment. The $\mathrm{B}$ and C fragments, sized 449 and $207 \mathrm{~kb}$, respectively, contain several enhancers and two of them, MACS1 (red ellipse) and ZRS (blue

\section{Combined $\mathrm{Hi}-\mathrm{C}$ and genome sequencing helps to resolve and interpret the complex rearrangement}

To understand the 3D genome landscape at the $\mathrm{SHH}$ locus, we analyzed fibroblasts $\mathrm{Hi}-\mathrm{C}$ maps from an unrelated healthy control (Melo et al. 2020) and we observed two topologically associating domains (TADs) at this locus: (i) the SHH-TAD which contains the $S H H, R N F 32$ and $L M B R 1$ genes, (ii) a telomeric TAD comprising NOM1, MNX1, UBE3C, and DNAJB6 (Fig. 4a and b). Importantly, sonic hedgehog (Shh) is a major developmental gene that controls cell survival and fate, and axial patterning in the vertebrate body plan. As shown in mouse mutants, $S h h$ is required for the growth and differentiation of the esophagus, trachea and lung (Litingtung et al. 1998). The SHH chromatin domain (cen-TAD) contains several known cis-regulatory elements (CREs) that control diverse expression patterns, including MRCS1, MFCS4, and MACS1, which drive Shh expression in the epithelia of the larynx, lung and intestinal and urogenital tracts (Fig. S4a; Sagai et al. 2009). MACS1, MRCS1 and MFCS4 as well as the limb enhancer ZRS are located in the SHH-TAD and are physically insulated from the telomeric TAD by a boundary element.

Next, we performed Hi-C in fibroblasts of Fetus 2 (III-3) and the father (II-1) to solve the nested structure of the SV and simultaneously interpret the 3D genome architecture of this region. Hi-C revealed a similar ectopic chromatin interaction pattern in the father and the fetus (Fig. $4 \mathrm{c}$ and d; Figs. S4b and S4c), however, the pattern signal was weaker in the father than in the fetus, indicating a lower copy number of the rearranged fragments, in accordance with his ellipse), regulate $S H H$ in a spatio-temporal manner. The other known enhancers are represented by orange ellipses. b Genome sequencing breakpoint analysis disclosed two possible linear sequence scenarios. Three interactions are observed by manual inspection of split-reads: D-A (1), C-B (2) and B-E' (3)

mosaicism detected previously. Noteworthy, a wild-type copy of MACS1 is still located within the SHH-TAD, thus SHH seems to be unaffected by position effect of this SV.

The A and D fragments have 15 and $9.8 \mathrm{~kb}$ in length, respectively, and do not contain known enhancers. Also the $\mathrm{E}$ fragment ( $61 \mathrm{~kb}$ heterozygous deletion) is devoid of known cis-regulatory elements (Fig. 4b). The B and C fragments, sized 449 and $207 \mathrm{~kb}$, respectively, contain several enhancers and two of them, MACS1 and ZRS, regulate $S H H$ (Fig. 4b; Anderson et al. 2014). Based on these findings, we then hypothesized that a novel enhancer-promoter interaction caused by the repositioning of the lung enhancers into another TAD might lead to gene misexpression during primary lung buds development, likely causing the disease.

To better visualize the impact of the SV in the Hi-C map, we subtracted the Hi-C signal of the control and we observed a strong chromatin interaction of about $680 \mathrm{~kb}$ in the fetus (Fig. 4d). After subtraction, the direct contact of Fragments B-E' and the $61 \mathrm{~kb}$ deletion (Fragment E) became visible. The simplest way of visualizing the derivative locus is drawing a schematic representation of the derivative landscape (Melo et al. 2020). Combining the linear sequence obtained from the GS data and adding on top the Hi-C information, we observed the formation of two new domains (neo-TADs) each about $\sim 670-680 \mathrm{~kb}$ in size. In both neo-TADs, the MACS1 enhancer can physically interact with $M N X 1$ and $N O M 1$ (Fig. 4e). Interestingly, $M N X 1$ is known to upregulate Wnt/B-catenin signaling and its downstream targets c-Myc and CCND1 (Yang et al. 2019).

Taken together, these data suggest that the $7 \mathrm{q} 36.3 \mathrm{SV}$ here identified creates two neo-TADs, thus allowing ectopic 


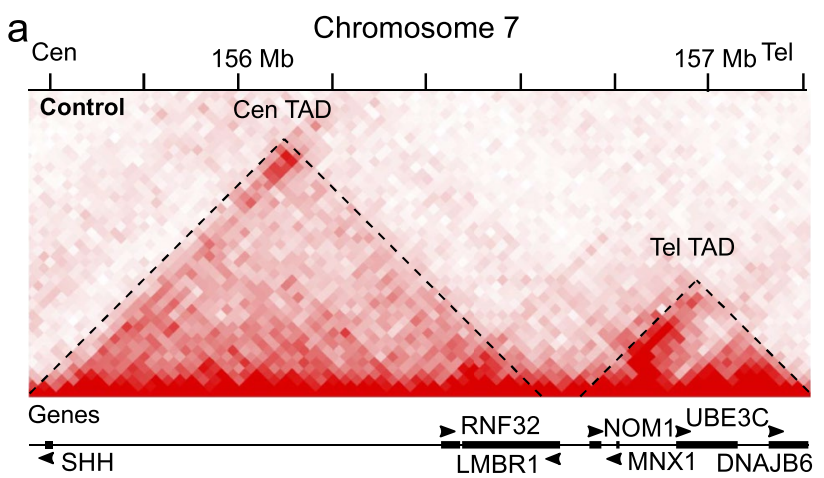

b
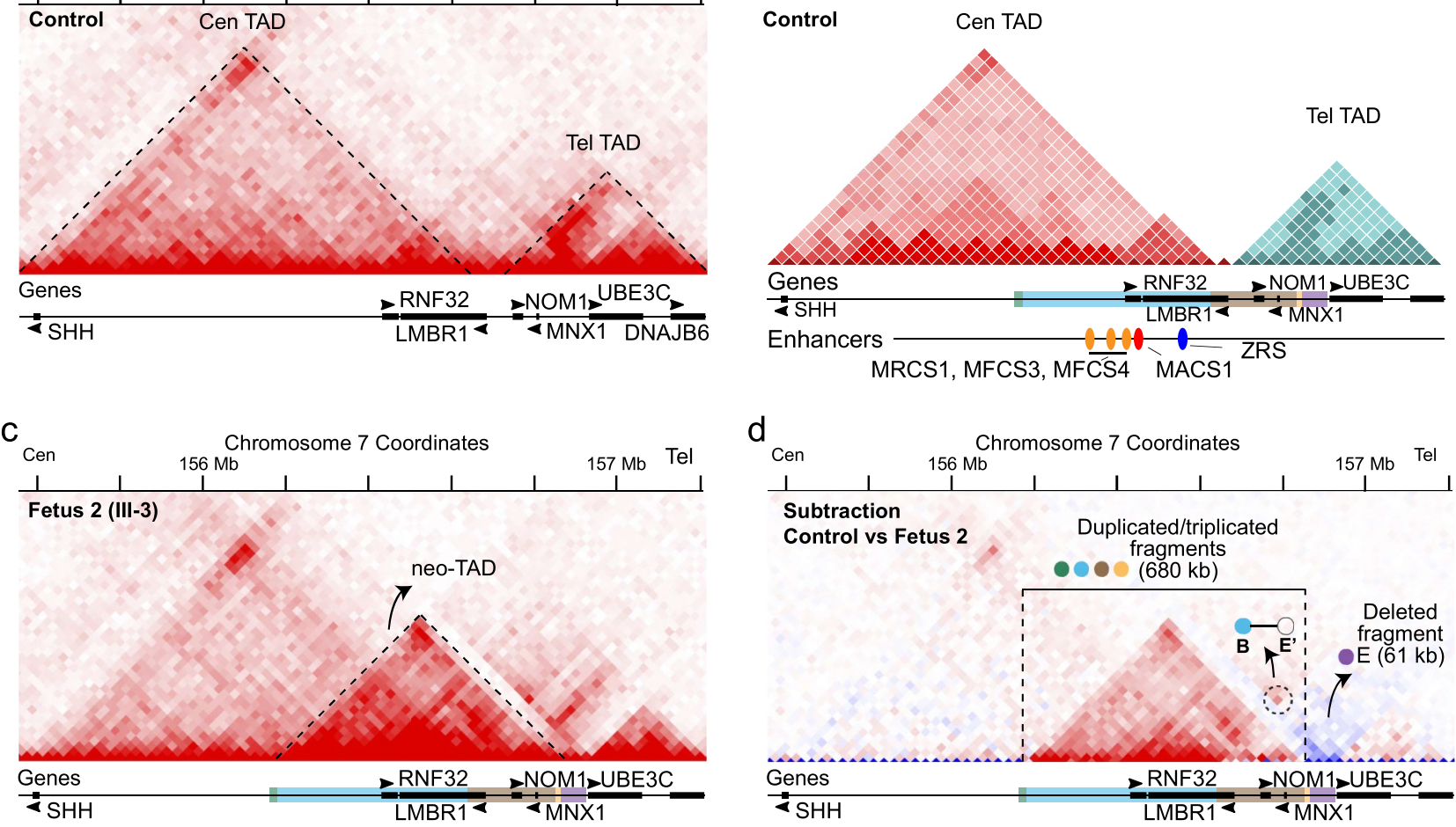

e

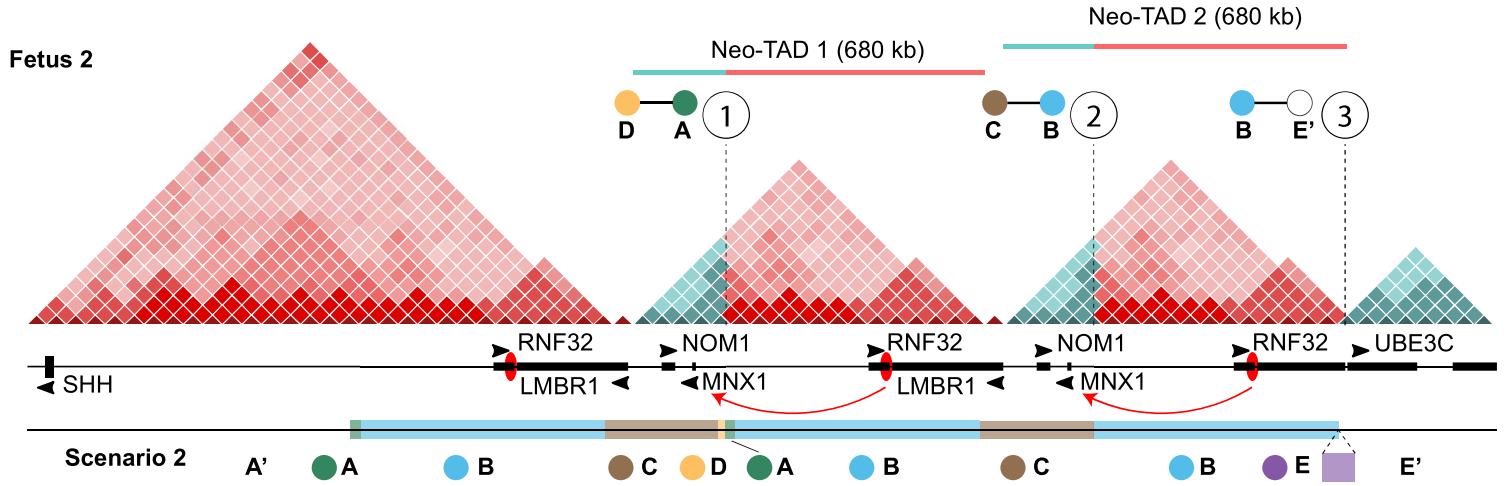

Fig. 4 3D chromatin landscape at the $\mathrm{SHH}$ locus in healthy and affected fibroblasts. a Hi-C map of control fibroblast sample $(25 \mathrm{~kb}$ resolution; raw data) showing the 3D landscape of the 7q36.3 locus. Genes (black rectangles; arrows indicate the orientation of the transcript) are listed below. b Schematic representation of TAD structures on the $7 \mathrm{q} 36.3$ region in a wild-type sample (the centromeric TAD in red and the telomeric one in blue). MACS1 and ZRS enhancers (colored in red and blue, respectively) are shown on the track below. Additional known oral and pharyngeal epithelium enhancers are shown in orange. c Hi-C map from fibroblasts the Fetus 2 revealed ectopic signal due to novel chromatin contacts. d Subtracted map

MACS1 contact with $M N X 1$ and NOM1 promoters in lung tissue, with a likely blocking effect on the WNT signaling pathway, resulting in absent development of primary air cells and eventually leading to the bilateral lung agenesis observed in the three fetuses. shows the gain of new chromatin interaction in the fetus. Red: gain of contact, blue: loss of contact. e Schematic representation of the derivative $7 \mathrm{q} 36.3$ 3D structure caused by the complex genomic rearrangement. Observe the formation of two neo-TADs, both allowing ectopic interaction of MACS1 with $M N X 1$ and NOM1 promoters (red arrows). Based on our analysis, both scenarios 1 and 2 are compatible to be true, i.e., they would give roughly similar outcomes related to 3D genome architecture reconstruction; therefore both are likely for being causative. In this work, we choose Scenario 2 for merely illustration purpose

\section{Discussion}

Complete absence of the lung is an ultra-rare malformation described in only few cases worldwide. Pulmonary hypoplasia is more common and mostly occurs in association 
with fetal hydrops, congenital diaphragmatic hernia, oligohydramnios, or skeletal dysplasia; a broader phenotypic spectrum can also be observed and includes patients harboring coding and non-coding pathogenic variants (Reviewed in Nogee and Ryan 2021). Rare familial occurrence has been described (Anderson et al. 2014). In the present study, the exceptional recurrence of complete bilateral lung agenesis in three fetuses suggested a genetic basis. After applying a combination of several genetic/genomic screening methodologies, we identified a CGR on $7 \mathrm{q} 36.3$ shared by all three affected fetuses, presenting duplicated, triplicated and deleted fragments. Previous studies have shown that accurate detection and interpretation of complex rearrangements should be done by integration of different genomic technologies (Carvalho and Lupski 2016); indeed, the complexity of this SV was only resolved by a combined strategy consisting of array CGH, GS, and Hi-C. Our study further supports the utility of $\mathrm{Hi}-\mathrm{C}$ for the detection, characterization and interpretation of complex genomic rearrangements. Hi-C was instrumental in the precise localization of the individual $\mathrm{CNVs}$, but was also helpful in interpreting the effects of the rearrangements.

The pedigree of the enrolled family in this work was suggestive of autosomal recessive inheritance for the lung phenotype, but ES in the affected fetuses failed to identify mono- or biallelic rare variants in known genes related to agenesis, aplasia or hypoplasia of the lung, or other potential candidate genes (Park et al. 2019; Arman et al. 1999; McPherson et al. 2009; Wang et al. 2006; Boucherat et al. 2014). The candidate CGR identified here was present in the unaffected father, first resulting in its interpretation as a variant of unknown significance. Incomplete penetrance or mosaicism was considered as another possibility. Based on several experiments including array CGH, qPCR, RT-qPCR, $\mathrm{GS}$ and $\mathrm{Hi}-\mathrm{C}$, we were able to conclude that the father is mosaic for this SV in agreement with the observed inheritance pattern. Thus, the careful dissection of this complex rearrangement resulted in an explanation completely different from our original hypothesis.

SVs can interfere with chromatin folding if they disrupt TADs. TADs have been identified as regions in the genome that show high interaction in the 3D space of the nucleus (Rao et al. 2014). They are separated from each other by regions of low interaction, so called boundaries. The interactions measured by chromosome conformation capture (3C) methods such as $\mathrm{Hi}-\mathrm{C}$ reflect the physical proximity of enhancers with the target promoters. Since Hi-C is a quantitative measure of proximity, it can also be used to identify rearrangements (Melo et al. 2020). Here, Hi-C together with array CGH and GS indicated a triplication of a region containing part of the SHH-TAD and thus $\mathrm{SHH}$ enhancers (B fragment) and a duplication of the boundary between the SHH-TAD and its neighboring TAD that contains the MNXI and NOMI genes (C fragment). As previously shown by us (Franke et al. 2016; Spielmann et al. 2018), duplications can result in the formation of novel chromatin domains, so called neo-TADs, if a boundary is included in the duplication. Therefore, regulatory elements are connected in the neo-TAD with genes that were previously separated. In the current case, the inclusion of the boundary suggests the generation of two neo-TADs each containing $\mathrm{SHH}$ enhancers and the $M N X 1$ and NOMI genes. However, we cannot exclude the involvement of other yet so far unknown lung enhancers in the region.

Sonic hedgehog (SHH) and its downstream effector GLIs are major players in determination of the fate of pulmonary bud cells (Warburton et al. 2005; Fernandes-Silva et al. 2017). Shh is widely expressed in the foregut endoderm and is specifically upregulated in the distal epithelium of the lung where branching is occurring (Miller et al. 2004). In Shh null mutants, the lungs form a rudimentary sac due to a failure of branching and growth after formation of the primary lung buds (Litingtung et al. 1998; Pepicelli et al. 1998). Furthermore, the deletion of two transcription factors mediating the Shh pathway, Gli2 and Gli3, resulted in an absent formation of lung, trachea and esophagus in mice (Motoyama et al. 1998), further supporting the importance of this pathway in lung development. It is thus to be expected that the SHH-TAD contains enhancers that drive Shh expression during lung morphogenesis, and a cluster of such enhancers has been identified of which MACS1 has been shown to be essential for Shh expression in the laryngeal epithelia and lung buds in mice (Sagai et al. 2009; Sagai et al. 2017). Accordingly, MACS1 knockouts show defects in the respiratory organogenesis (Sagai et al. 2017). A more common finding than lung aplasia are tracheal malformations, sometimes in combination with tracheoesophageal fistulas (Evans et al. 1999). These malformations are observed in the well-described VATER association. After experimental induction of tracheoesophageal malformations in an animal model a dysregulation of $\mathrm{SHH}$ was found (Ioannides et al. 2003). Interestingly, a deletion of 7q35q36.3 was found in a case with VATER symptoms (PMID 27436264). It would be further interesting to perform Hi-C in such cases to learn more about the impact of the rearrangements on the $\mathrm{SHH}$ locus. It is also known that $\mathrm{SHH}$ mutations are associated with other congenital malformations, e.g., Holoprosencephaly 3 (MIM 142945), Microphthalmia with coloboma 5 (MIM 611638), Single median maxillary central incisor (MIM 147250), and Schizencephaly (MIM 269160), however, as expected, the symptoms detected in these disorders were not present/evaluated in our fetuses due to the preservation of the wild-type SHH-TAD.

The other TAD involved in the duplications contains $M N X 1$, also called HLXB9, encoding a homeobox transcription factor expressed in lymphocytes, colon, stomach, 
small Intestine, pancreas, and pituitary gland (Harrison et al. 1999). Heterozygous mutations in $M N X 1$ cause Currarino triad (MIM \#176450; Lynch et al. 1995; Ross et al. 1998), a condition characterized by partial sacral agenesis, a presacral mass, and anorectal malformation. Here we hypothesize that ectopic activation of this gene by the MACS1 or other lung enhancers could have catastrophic effects in the fine-tuned network of signaling molecules necessary for proper lung development. MNX1 is known to upregulate $\mathrm{Wnt} / \mathrm{B}$-catenin signaling and its downstream genes c-Myc and CCND1. An alteration of this pathway could lead to a failure in formation or outgrowth of the primary lung buds (Yang et al. 2019). Additionally, the long non-coding RNA (lncRNA) MNX1-AS1 activates MAPK signaling, another important pathway which may be involved in proper lung development (Liu et al. 2019), for the reason that Mekl/Mek2 double knockout presents pulmonary hypoplasia and other related phenotypes (Boucherat et al. 2014). A similar mechanism has been described for Acropectorovertebral syndrome (OMIM: \#102510) in which misexpression of PAX3 under the control of EPHA4 enhancers causes a complex limb malformation (Lupiáñez et al. 2015). However, additional studies need to be performed to support the hypothesis of MNXI activation by MACS1 in lung tissue as the cause of lung agenesis in our patients.

In conclusion, the current observation provides the first example of congenital absence of lungs likely due to a TAD disorganization by a copy number variation. It further substantiates the importance of proper interpretation of CNVs above their positional constrains to elucidate the missing heritability. Thus, ectopic enhancer-promoter interaction could lead to $M N X 1$ activation in lung cells by MACS1, causing the lung agenesis in the three fetuses.

Supplementary Information The online version contains supplementary material available at https://doi.org/10.1007/s00439-021-02344-6.

Acknowledgements We thank the family for its collaboration to this work. Funding: S.M. is supported by grants from the Deutsche Forschungsgemeinschaft (DFG) SP1532/3-1, SP1532/4-1, SP1532/5-1, and MU 880/16-1) and the Max Planck Foundation. F.L.H. was supported by the Research Promotion Fund of the Faculty of Medicine (FFM) of the University Medical Center Hamburg-Eppendorf and by a grant from the Deutsche Forschungsgemeinschaft (KU 1240/10-1). M.A.M. is a participant in the BIH Charité Digital Clinician Scientist Program founded by the late Prof. Duska Dragun and funded by the Charité-Universitätsmedizin Berlin and the Berlin Institute of Health.

Author contributions Conceptualization: USM, JP, BF-Z, SM, LVM. Funding acquisition: SM, LVM. Methodology: USM, M-KK, RS, MAM, MH, FAG, AM, VG, DG, EL, VR, VK, RR, CC, FLH, MS. Project administration: USM, UK, SM, LVM. Resources: SM, LVM. Validation: USM, JP, M-KK, RS. Visualization: USM. Writing-original draft: USM, JP. Writing — review and editing: USM, JP, BF-Z, SM, LVM.
Funding Open Access funding enabled and organized by Projekt DEAL.

Data availability Data and materials are available upon request.

\section{Declarations}

Conflict of interest The authors declare no competing interests.

Ethics declaration This study was approved by the review board of the National Institute of Health and Medical Research (INSERM), CHU, Besançon, France. Healthy parents provided written informed consent to all subjects enrolled in this study giving permission to use DNA samples for genomic screening, (2) skin fibroblasts for establishing cell lines, and (3) photos for research publications, in accordance with the rules of Helsinki.

Open Access This article is licensed under a Creative Commons Attribution 4.0 International License, which permits use, sharing, adaptation, distribution and reproduction in any medium or format, as long as you give appropriate credit to the original author(s) and the source, provide a link to the Creative Commons licence, and indicate if changes were made. The images or other third party material in this article are included in the article's Creative Commons licence, unless indicated otherwise in a credit line to the material. If material is not included in the article's Creative Commons licence and your intended use is not permitted by statutory regulation or exceeds the permitted use, you will need to obtain permission directly from the copyright holder. To view a copy of this licence, visit http://creativecommons.org/licenses/by/4.0/.

\section{References}

Anderson E, Devenney PS, Hill RE, Lettice LA (2014) Mapping the Shh long-range regulatory domain. Development 141(20):3934-3943

Arman E, Haffner-Krausz R, Gorivodsky M, Lonai P (1999) Fgfr2 is required for limb outgrowth and lung-branching morphogenesis. Proc Natl Acad Sci U S A 96(21):11895-11899. https://doi. org/10.1073/pnas.96.21.11895 (PMID: 10518547; PMCID: PMC18383)

Boucherat O, Nadeau V, Bérubé-Simard FA, Charron J, Jeannotte L (2014) Crucial requirement of ERK/MAPK signaling in respiratory tract development. Development 141(16):3197-3211. https:// doi.org/10.1242/dev.110254 (Erratum. In: Development.2015 Nov 1;142(21):3801)

Carvalho CM, Lupski JR (2016) Mechanisms underlying structural variant formation in genomic disorders. Nat Rev Genet 17(4):224 238. https://doi.org/10.1038/nrg.2015.25 (Epub 2016 Feb 29. PMID: 26924765; PMCID: PMC4827625)

Chiang C, Litingtung Y, Lee E, Young KE, Corden JL, Westphal H et al (1996) Cyclopia and defective axial patterning in mice lacking Sonic hedgehog gene function. Nature 383:407-413

Durand NC, Shamim MS, Machol I, Rao SSP, Huntley MH, Lander ES, Aiden EL (2016) Juicer provides a one-click system for analyzing loop-resolution Hi-C experiments. Cell Syst 3:95-98

Engellenner W, Kaplan C, Van de Vegte GL (1989) Pulmonary agenesis association with nonimmune hydrops. Pediatr Pathol 9:725-730

Evans JA, Greenberg CR, Erdile L (1999) Tracheal agenesis revisited: analysis of associated anomalies. Am J Med Genet 82(5):415-422

Fernandes-Silva H, Correia-Pinto J, Moura RS (2017) Canonical Sonic hedgehog signaling in early lung development. J Dev Biol 5(1):3 
Franke M et al (2016) Formation of new chromatin domains determines pathogenicity of genomic duplications. Nature 538(7624):265-269

Harrison KA, Thaler J, Pfaff SL, Gu H, Kehrl JH (1999) Pancreas dorsal lobe agenesis and abnormal islets of Langerhans in Hlxb9deficient mice. Nat Genet 23(1):71-75

Ioannides AS, Henderson DJ, Spitz L, Copp AJ (2003) Role of Sonic hedgehog in the development of the trachea and oesophagus. J Pediatr Surg 38(1):29-36

Kadzik RS, Morrisey EE (2012) Directing lung endoderm differentiation in pluripotent stem cells. Cell Stem Cell 10(4):355-361

Kayemba-Kay's S, Couvrat-Carcauzon V, Goua V, Podevin G, Marteau M, Sapin E, Levard G (2014) Unilateral pulmonary agenesis: a report of four cases, two diagnosed antenatally and literature review. Pediatr Pulmonol 49(3):E96-102

Kimura J, Deutsch GH (2007) Key mechanisms of early lung development. Pediatr Dev Pathol 10(5):335-347

Litingtung Y, Lei L, Westphal H, Chiang C (1998) Sonic hedgehog is essential to foregut development. Nat Genet 20(1):58-61

Liu X, Yang Q, Yan J, Zhang X, Zheng M (2019) LncRNA MNX1-AS1 promotes the progression of cervical cancer through activating MAPK pathway. J Cell Biochem 120(3):4268-4277

Lupiáñez DG et al (2015) Disruptions of topological chromatin domains cause pathogenic rewiring of gene-enhancer interactions. Cell 161(5):1012-1025

Lynch SA, Bond PM, Copp AJ, Kirwan WO, Nour S, Balling R, Mariman E, Burn J, Strachan T (1995) A gene for autosomal dominant sacral agenesis maps to the holoprosencephaly region at $7 \mathrm{q} 36$. Nat Genet 11:93-95

Mardini MK, Nyhan WL (1985) Agenesis of the lung. Report of four patients with unusual anomalies. Chest 87:522-527

McPherson JP, Sarras H, Lemmers B, Tamblyn L, Migon E, MatysiakZablocki E, Hakem A, Azami SA, Cardoso R, Fish J, Sanchez O, Post M, Hakem R (2009) Essential role for Bclaf1 in lung development and immune system function. Cell Death Differ $16(2): 331-339$

Melo US et al (2020) Hi-C identifies complex genomic rearrangements and TAD-shuffling in developmental diseases. Am J Hum Genet 106(6):872-884

Miller LA et al (2004) Role of sonic hedgehog in patterning of trachealbronchial cartilage and the peripheral lung. Dev Dyn 231:57-71

Motoyama J, Liu J, Mo R, Ding Q, Post M, Hui CC (1998) Essential function of Gli2 and Gli3 in the formation of lung, trachea and oesophagus. Nat Genet 20(1):54-57

Nogee LM, Ryan RM (2021) Genetic testing for neonatal respiratory disease. Children (basel) 8(3):216. https://doi.org/10.3390/child ren8030216 (PMID: 33799761; PMCID: PMC8001923)

Ostör AG, Stillwell R, Fortune DW (1978) Bilateral pulmonary agenesis. Pathology 10(3):243-248

Park J, Ivey MJ, Deana Y, Riggsbee KL, Sörensen E, Schwabl V, Sjöberg C, Hjertberg T, Park GY, Swonger JM, Rosengreen T, Morty
RE, Ahlbrecht K, Tallquist MD (2019) The Tcf21 lineage constitutes the lung lipofibroblast population. Am J Physiol Lung Cell Mol Physiol 316(5):L872-L885

Pepicelli CV, Lewis PM, McMahon AP (1998) Sonic hedgehog regulates branching morphogenesis in the mammalian lung. Curr Biol 8:1083-1086

Podlech J, Richter J, Czygan P, Klein J, Mûntefering H (1995) Bilateral agenesis/aplasia of the lungs: report of a second case in the offspring of one women. Pediatr Pathol Lab Med 15:781-790

Rao SSP et al (2014) A 3D map of the human genome at kilobase resolution reveals principles of chromatin looping. Cell 159:1665-1680

Ross AJ et al (1998) A homeobox gene, HLXB9, is the major locus for dominantly inherited sacral agenesis. Nat Genet 20:358-361

Sagai T, Amano T, Tamura M, Mizushina Y, Sumiyama K, Shiroishi T (2009) A cluster of three long-range enhancers directs regional SHH expression in the epithelial linings. Development 136(10):1665-1674

Sagai T et al (2017) Evolution of Shh endoderm enhancers during morphological transition from ventral lungs to dorsal gas bladder. Nat Commun 8:14300

Schittny JC (2017) Development of the lung. Cell Tissue Res 367(3):427-444

Schmittgen TD, Livak KJ (2008) Analyzing real-time PCR data by the comparative C(T) method. Nat Protoc 3(6):1101-1108

Spear GS, Yetur P, Beyerlein RA (1987) Bilateral pulmonary agenesis and microphthalmia. Am J Med Genet Suppl 3:379-382

Spielmann M, Lupianez DG, Mundlos S (2018) Structural variation in the 3D genome. Nat Rev Genet 19:455-465

Swarr DT, Morrisey EE (2015) Lung endoderm morphogenesis: gasping for form and function. Annu Rev Cell Dev Biol 31:553-573

van Baren MJ, van der Linde HC, Breedveld GJ, Baarends WM, Rizzu P, de Graaff E, Oostra BA, Heutink P (2002) A double RING$\mathrm{H} 2$ domain in RNF32, a gene expressed during sperm formation. Biochem Biophys Res Commun 292(1):58-65

Wang Z, Dollé P, Cardoso WV, Niederreither K (2006) Retinoic acid regulates morphogenesis and patterning of posterior foregut derivatives. Dev Biol 297(2):433-445

Warburton D, Bellusci S, De Langhe S, Del Moral PM, Fleury V, Mailleux A, Tefft D, Unbekandt M, Wang K, Shi W (2005) Molecular mechanisms of early lung specification and branching morphogenesis. Pediatr Res 57(5 Pt 2):26R-37R

Yang X, Pan Q, Lu Y, Jiang X, Zhang S, Wu J (2019) MNX1 promotes cell proliferation and activates $\mathrm{Wnt} / \beta$-catenin signaling in colorectal cancer. Cell Biol Int 43(4):402-408

Publisher's Note Springer Nature remains neutral with regard to jurisdictional claims in published maps and institutional affiliations. 


\section{Authors and Affiliations}

Uirá Souto Melo ${ }^{1,2} \cdot$ Juliette Piard $^{3} \cdot$ Björn Fischer-Zirnsak ${ }^{2} \cdot$ Marius-Konstantin Klever $^{1,2} \cdot$ Robert Schöpflin $^{1}$. Martin Atta Mensah ${ }^{2,4} \cdot$ Manuel Holtgrewe ${ }^{4}$. Francine Arbez-Gindre ${ }^{5}$. Alain Martin ${ }^{5}$. Virginie Guigue ${ }^{5,6}$.

Dominique Gaillard $^{6}$. Emilie Landais ${ }^{6} \cdot$ Virginie Roze $^{5} \cdot$ Valerie Kremer $^{7,8,13} \cdot$ Rajeev Ramanah $^{5} \cdot$ Christelle Cabrol $^{3}$. Frederike L. Harms ${ }^{9} \cdot$ Uwe Kornak $^{10}$. Malte Spielmann ${ }^{1,11}$. Stefan Mundlos ${ }^{1,2}$. Lionel Van Maldergem ${ }^{3,4,12}$

1 Max Planck Institute for Molecular Genetics, RG Development and Disease, Berlin, Germany

2 Institute of Medical Genetics and Human Genetics, Charité-Universitätsmedizin Berlin, Corporate Member of Freie Universität Berlin and Humboldt-Universität zu Berlin, Berlin, Germany

3 Centre de Génétique Humaine, Université de Franche-Comté, Besançon, France

4 Berlin Institute of Health at Charité-Universitätsmedizin Berlin, Berlin, Germany

5 Departments of Obstetrics and Gynecology, Pathology, and Biology, University Hospital, University of Franche-Comte, Besançon, France

6 Department of Obstetrics and Gynecology, University Hospital Grenoble-Alpes, Grenoble, France

7 Department of Medical Genetics, University Hospital, University Champagne-Ardennes, Reims, France
8 Laboratory of Cytogenetics, University Hospital, University of Strasbourg, Strasbourg, France

9 Institute of Human Genetics, University Medical Center Hamburg-Eppendorf, Hamburg, Germany

10 Institute of Human Genetics, Universitätsmedizin Göttingen, Göttingen, Germany

11 Institute of Human Genetics, University of Lübeck, Lübeck, Germany

12 Center of Clinical Investigation (CIC), National Institute of Health and Medical Research (INSERM), CHU, Besançon, France

13 Present Address: Department of Medical Genetics, University Hospital, Geneva, Switzerland 